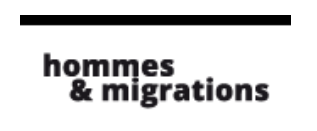

\section{Hommes \& migrations}

Revue française de référence sur les dynamiques

migratoires

$1283 \mid 2010$

Cuisines et dépendances

\title{
Migrations et pratiques culinaires
}

Avenir de la cuisine endogène et santé des populations en milieu urbain au Cameroun

Jean-Marie Essomba, Mbonji Edjenguèlè, Patrick Pasquet et Annie Hubert

\section{OpenEdition}

\section{Journals}

Édition électronique

URL : http://journals.openedition.org/hommesmigrations/1002

DOI : 10.4000/hommesmigrations.1002

ISSN : 2262-3353

Éditeur

Musée national de l'histoire de l'immigration

Édition imprimée

Date de publication : 1 janvier 2010

Pagination : 136-149

ISBN : 978-2-919040-04-9

ISSN : $1142-852 X$

Référence électronique

Jean-Marie Essomba, Mbonji Edjenguèlè, Patrick Pasquet et Annie Hubert, « Migrations et pratiques culinaires », Hommes \& migrations [En ligne], 1283 | 2010, mis en ligne le 29 mai 2013, consulté le 19 avril 2019. URL : http://journals.openedition.org/hommesmigrations/1002 ; DOI : 10.4000/ hommesmigrations.1002 


\section{Migrations et pratiques culinaires \\ Avenir de la cuisine endogène et santé des populations en milieu urbain au Cameroun}

Par Jean Marie Essomba, anthropologue, chercheur au département d'anthropologie, université de Yaoundé I, Cameroun, Mbonji Edjenguèlè, anthropologue, professeur, université de Yaoundé I, Cameroun,

Patrick Pasquet, anthropologue, directeur de recherche au CNRS et enseignant au MNHN, Paris

Annie Hubert, anthropologue, directeur de recherche au CNRS, Paris

La cuisine, telle qu'elle est pratiquée dans les villes africaines au sud du Sahara, inquiète ses consommateurs. À Yaoundé, les migrants béti et bamiléké s'efforcent d'entretenir l'identité de leur cuisine, tout en faisant face aux dangers pour la santé des nourritures issues des standards de la modernité : trop d'épices, de calories, de gras.

Mais cet équilibre lui-même est menacé par manque de temps et du fait de la disparition de certains ingrédients ou de savoir-faire. 
Dans toutes les cultures, il existe un terme pour désigner la cuisine, et sa définition est presque toujours le même : la transformation des aliments dans le but de les rendre consommables. Mais la cuisine est aussi un lieu, ou mieux encore, un socioespace où se réalisent ces transformations. Ce socio-espace qu'est la cuisine fait partie d'un lieu de, et ce dans toutes les cultures humaines, comme le soulignent Annie Hubert et Geneviève Cazes-Valette ${ }^{(1)}$. Dans toutes les cultures, la cuisine a une mission : rendre comestible ce qui, au départ, est mangeable. Mais la modernisation des modes de vie semble nous conduire à la rencontre entre la culture culinaire moderne et la cuisine traditionnelle. La perte des repères dans le rythme alimentaire familial devient petit à petit un facteur de nature à fragiliser la construction des comportements alimentaires normaux et favorisant le développement de troubles du comportement alimentaire. Avec l'urbanisation et les décalages des horaires de travail en milieu urbain au Cameroun, on assiste à une déstructuration des recettes et des manières de faire pour intégrer, dans les pratiques traditionnelles, les apports des nouvelles technologies et des nouvelles perceptions des nourritures et de la cuisine. Dans ce double attachement à la cuisine moderne et traditionnelle, comment font les migrants pour gérer culturellement les transitions culinaires, afin de s'intégrer à un nouveau contexte culinaire sans se couper du passé réel ou mythique? Cette gestion de la cuisine moderne et traditionnelle milite-t-elle pour une cuisine d'héritage et/ou l'homo-cuisine? Peut-elle favoriser le développement de l'hétéro-cuisine? Ces trois questions constituent les grandes articulations de notre réflexion.

\section{Code culinaire en milieu urbain au Cameroun : de l'homo-cuisine à l'hétéro-cuisine}

Si nous considérons la cuisine sous une approche matérielle, nous pouvons admettre que la simplicité de sa composition se retrouve partout dans le monde. François Sigaut ne s'éloigne pas de cette perception lorsqu'il affirme: “ À quelques exceptions près, tous les peuples de la terre composent leurs repas ordinaires de la même façon : un aliment de base, qui apporte l'essentiel de ce qui est considéré comme 'nourriture' proprement dite, et un accompagnement fait d'ingrédients variés (viande, poisson, laitage, légumes, condiments, etc.) destiné à donner du goût, à 'faire passer' l'aliment de base, souvent assez fade $e^{(2)}$." Il n'est pas rare que cet accompagnement porte un nom spécifique : "relish", dans l'anglais parlé en Afrique noire. Pour donner une idée de ce que donne cette formule "nourriture + sauce" lorsqu'elle est mise pratique, il suffit de citer, par exemple, les spaghettis à la sardine ou à l'avocat. 
Un féculent pour nourriture, dont la nature pour l'instant nous importe peu, et une sauce pour faire passer le féculent: voici la structure des repas depuis des milliers d'années.

Dans le domaine de la cuisine, la réflexion ci-dessus est l'illustration d'une formule universelle à laquelle le Cameroun en général et les migrants en particulier ne dérogent pas. Chez les migrants béti, par exemple, nous retrouvons le manioc accompagné de la sauce d'arachide ou des feuilles de manioc, alors que chez les migrants bamiléké, le taro est accompagné de la sauce jaune. Bien que cette spécificité matérielle soit une constante, on la retrouve tout de même sous des aspects différents. En effet, le modèle de préparation "nourriture+sauce" change de manière permanente, tant par la nature de la préparation des féculents que par celle des sauces. Les brisures de riz, aujourd'hui très présentes dans la cuisine des migrants béti et bamiléké, et surtout dans la restauration ambulante, ne relèvent que d'une innovation relativement récente qui remonte à la fin du $\mathrm{XX}^{\mathrm{e}}$ siècle. En ce qui concerne la sauce, le concentré de tomate et le cube Maggi ont également conquis, en quelques années, leur droit de cité dans la cuisine des migrants.

Ce qui précède conduit à deux observations importantes. Dans un premier temps, les pratiques culinaires des migrants n'évoluent pas vers des pratiques culinaires occidentales, comme semblent le dire les spécialistes de la théorie du mimétisme. Au contraire, pour les populations urbaines, la recherche d'une alimentation plus variée ne saurait contribuer à la disparition de la cuisine du fait d'un supposé mimétisme. Mais la ville semble plutôt mal adaptée aux nouvelles conditions de la demande. Une conclusion confirmée par cet article montre bien, de bout en bout, que, les migrants béti et bamiléké cuisinent camerounais, même s'ils incorporent dans leurs ethnocodes d'autres éléments venant de l'extérieur, comme cela arrive dans toutes les cuisines du monde.

Dans un second temps, la cuisine traditionnelle est confrontée à la cuisine urbaine. Les deux véhiculent des représentations chargées de valeurs symboliques spécifiques à la culture de chaque migrant. Les plats bétiphones, tels que la sauce d'arachide, le kpwem, le nnam wondo, etc., ne sont plus les mêmes lorsqu'ils sont préparés en ville même par les Béti et, plus encore, par les Bamiléké.

Il en est de même pour la préparation des plats bamiléké comme le koki, le pilé, la sauce jaune. En fait, les plats, qu'ils soient préparés par les migrants béti ou bamiléké, ont une chose en commun : ils ne sont pas des "atomes isolés", pour reprendre l'expression de Syfia, parlant de produits alimentaires ${ }^{(3)}$. En effet, les plats, les mets, sont en relation avec d'autres mets et plats, eux aussi chargés d'une valeur symbolique. De même, ils sont en relation avec les hommes qui les préparent et les mangent, avec leurs croyances et leurs ethno-codes. 
Les ethno-codes culinaires représentent, l'ensemble des principes culturels internes en usage dans le domaine de la cuisine. Ils sont associés à un système de valeur, à une culture alimentaire endogène. Autrement dit, la cuisine, au même titre que le langage, est une forme d'expression : elle est constituée de signes, de sons et de sens, de dits et de non-dits, de paroles et de silences. La cuisine, comme le langage, évolue, mais pas de n'importe quelle façon. De ce point de vue, la cuisine en milieu urbain au Cameroun évolue aussi méthodiquement.

\section{Urbanisation et développement des recettes : le temps, l'espace et la pluralité}

Généralement, les recettes se combinent de façon différente, et ceci selon les cultures. Cette combinaison s'assimile à ce que Garine, Messer et Fischler appellent des "systèmes culinaires originaux ${ }^{(4)}$ ". Et l'ethnographie de l'alimentation des migrants béti et bamiléké nous révèle un exemple de pluralité de ces recettes "traditionnelles" (voir encadré). En effet, les deux groupes de migrants ont sélectionné dans leur environnement un grand nombre de substances qu'ils estiment comestibles. Seulement, toutes n'ont pas la même valeur symbolique et gustative.

L'ethnographie de l'alimentation des migrants béti et bamiléké montre un exemple de la diversité des cuisines "traditionnelles". Les Bamiléké viennent de l'ouest du Cameroun avec comme aliment de base le maïs. Alors que les Béti occupent la région du centre ayant pour aliment de base le manioc. Tous sont des cultivateurs

\section{Quelques recettes des Bamiléké préparées en ville}

Taha jhekouoo : on l'appelle en français, "Tenue militaire". Ce met se compose de maïs en farine, de sel, de feuilles de taro, d'eau, de piment, de crevettes et d'huile rouge. Le mélange est emballé dans les feuilles de bananier puis cuit.

Jhejha : composé du Koki (petit haricot appelé en français "niébé"), de piment, de sel et d'huile rouge. Le tout est emballé dans les feuilles de bananier.

Kouakekou : huile rouge, gombo, sel, piment Macabo râpé (Macabo xanthrosoma). Emballer dans les feuilles de bananier, laisser cuire le tout puis consommer avec de la sauce d'arachide au gombos (arachide, huile rouge, gombo, sel, piment).

Sack jho : couscous de maïs sec à la sauce de nkui - liquide gluant additionné de condiments écrasés : sesieho, ngachu, sena, melep, lemka, tchetcho'o, khenou, nzefe, diepse, gouack (sel), sou (piment), nse bap (eau de viande), kannwa (sel gemme).

Koki : plat fait à partir du niébé (vigna ungulata) avec de l'huile rouge, du sel et du piment. 


\section{Différentes recettes chez les Béti en ville}

Nkpwem : jeunes feuilles de manioc pilées, cuites à l'eau avec l'écorce d'olon . Quand les feuilles ont changé de couleur et d'odeur, on y ajoute le jus de noix de palme et on laisse bouillir jusqu'à obtention d'un mélange homogène.

Mbwet : bouillie de pâte de maïs frais au jus de canne à sucre. On écrase ou on pile de la canne à sucre pour en extraire le jus, puis on le mélange avec la pâte en y ajoutant de l'eau. Le tout est cuit au feu dans une marmite ouverte. On tourne avec une spatule en bois jusqu'à obtenir un mélange épais et homogène.

N'Fian wondo : sauce d'arachides. Dans de l'eau, on met du poisson séché préalablement nettoyé, du sel, des feuilles de basilic sauvage (ossim) écrasées, des épinards hachés et un peu d'huile de palme. On porte à ébullition. La pâte d'arachides est ensuite incorporée pour obtenir une sauce homogène légèrement épaisse.

Nnam ngoan : gâteau de pistaches. Faire cuire de la viande ou du poisson dans de l'eau, avec des oignons découpés et du sel. Retirer l'eau de cuisson de la viande ou du poison et y incorporer la pâte de pistaches pour obtenir un mélange très épais, y mettre les écrevisses, puis la viande. On emballe le tout dans des feuilles de bananier préalablement ramollies au feu et on fait cuire au bain-marie.

Nnam wondo : gâteau d'arachide (arachis hypogaea). On délaye de la pâte d'arachides dans de l'eau chaude pour obtenir un mélange homogène très épais, puis on ajoute le poisson séché déjà nettoyé. Le tout est emballé dans des feuilles de bananier déjà ramollies au feu et cuit au bain-marie.

Efirgui zoan : aubergines écrasées. Les aubergines sont légèrement cuites avec un peu d'eau salée, puis écrasées dans une marmite avec une spatule en bois. On ajoute de la pâte d'arachides. Quand le mélange posé sur le feu est bien épais, on y verse un peu d'huile de palme.

de tubercules, de céréales et de nombreuses autres espèces de plantes utilisées comme légumes. Ils ont sélectionné chacun dans leur environnement un grand nombre de substances qu'ils estiment comestibles. Mais toutes n'ont pas, comme nous le verrons, la même valeur symbolique ou gustative.

On repère dans la langue des deux groupes ethniques une classification de l'ensemble de la nourriture qui utilise le type de cuisson comme critère. Aussi peuton retrouver des aliments bouillis, frits, cuits à l'étouffé et, enfin, grillés l'utilisation du feu étant une originalité de l'alimentation des êtres de culture, selon le constat de Perlès ${ }^{(5)}$.

La cuisson semble jouer un rôle hautement symbolique dans les deux cultures des migrants. Cela rejoint ce que de nombreux anthropologues ont relevé dans leurs analyses concernant la structure culinaire ${ }^{(6)}$. Par conséquent, le contenu sémantique des plats est révélateur de cette structure culinaire. Celle-ci s'articule autour de la pluralité et du changement. La pluralité des mets et des préparations, mais 
aussi la diversité des recettes présentées, en est l'illustration illustration visible. Cependant, on note des similitudes dans la confection de certains mets entre les Béti et les Bamiléké ; c'est le cas, par exemple, des mets comme l'eba fon ou l'ekomba chez les Béti, qui s'apparente au taha piac chez les autres, ou l'essankan wondo qui ressemble au bouop piac des Bamiléké.

Mais il faut relever que beaucoup de différences existent entre les façons de préparer des Béti et des Bamiléké. En effet, si l'on rencontre beaucoup d'arachide dans la confection des mets béti, on parle très peu d'huile de palme. L'huile de palme est seulement de plus en plus consommée avec l'urbanisation, pour des raisons surtout économiques. Par contre, chez les Bamiléké, pratiquement tous les mets contiennent une grande quantité d'huile de palme, qui est dans la plupart des cas très peu ou pas du tout chauffée. Cette huile de palme a d'ailleurs une place importante dans leur culture, puisqu'elle est souvent exigée dans certaines cérémonies importantes comme le mariage.

Domba : poisson d'eau douce cuit à l'étouffée. On assaisonne le poisson nettoyé et coupé en morceaux avec les feuilles d'ossim, de messep (sorte de menthe) et d'odjom. Après ajout de sel et de piments écrasés, le tout est emballé dans des feuilles de bananier ramollies au feu et cuit au bain-marie.

Foass : larves d'hannetons. On nettoie les larves en évacuant le contenu de l'abdomen, puis on assaisonne avec du sel, de l'ossim, des oignons, du messep. À ébullition, on verse l'huile de l'adjap (baillonela toxisperma). On laisse cuire jusqu'à ce que les larves deviennent tendres et que l'eau soit complètement évaporée.

Eba fon ou Ekomba : pâte de maïs. On mélange de la pâte de maïs avec de la pâte d'arachides, puis des écrevisses. Le tout est emballé dans des feuilles de bananier préalablement ramollies, puis cuit au bain-marie.

Sa fono : on fait cuire des feuilles de manioc pilées avec du maïs sec précuit. On y ajoute le jus de noix de palme et on laisse cuire le tout.

Sanga : feuilles de morelle noire finement découpées, maïs frais et jus de noix de palme. Cuire la morelle et le maïs avec un morceau d'olon (écorce). Lorsque la morelle et le maïs sont cuits, ajouter le jus de noix de palme et continuer la cuisson jusqu'à ce que le mélange soit devenu épais.

Minkôn : chenilles marron clair (bombycomorpha) qu'on récolte sur l'Ayos. On fait griller les chenilles avec de la braise pour faire partir les poils. Elles sont ensuite lavées et posées sur le feu en assaisonnant avec du messep, de l'ossim, des piments, de l'huile et des oignons. On fait cuire jusqu'à que l'eau soit complètement évaporée.

Nfian essouk : sauce de jus de noix de palme. On assaisonne le jus de noix avec du messep, des piments et du sel, puis on le porte à ébullition. Après ajout de pâte d'arachide, on laisse cuire. 
Par ailleurs, les Bamiléké utilisent un large éventail de condiments faits à base de feuilles et d'écorces, alors que les Béti utilisent généralement des feuilles (messep, ossim) et des produits carnés pour relever le goût de leurs plats (écrevisses, poissons frais ou séchés, viande fraîche ou boucanée). Les Bamiléké ne consomment pas beaucoup de produits carnés.

Enfin, les Béti appellent "compléments" les denrées qui ne sont là que pour accompagner les plats (plantain, igname, macabo, manioc, taro...) et qui sont généralement cuits simplement à la vapeur. Mais, ces accompagnements se transforment en mets à part entière chez les Bamiléké ; en y ajoutant des condiments, de l'huile de palme, du sel, et, parfois, des feuilles pilées, elles deviennent un plat en soi.

\section{Attitudes des migrants béti et bamiléké}

Les Béti et les Bamiléké sont deux peuples qui ont tous les deux migré vers les villes. Mais, à y regarder de plus près, leur intégration à la vie urbaine s'est faite à deux vitesses. Dans les foyers béti, on est tout de suite frappé par le niveau de modernisation des ustensiles qui servent à la cuisine. Ils sont devenus modernes avec un degré de sophistication dépendant du niveau social. En effet, il est de plus en plus difficile de trouver des ustensiles comme des cuillères en bois. Le feu de bois est présent chez ceux qui sont vraiment démunis ou qui préparent rarement des mets typiquement traditionnels. Même les menus quotidiens privilégient les aliments qu'on a appris à consommer en ville, comme le riz, les pâtes alimentaires, les poissons de mer, la viande du bceuf. Dans ce moment de transition, seule l'huile d'arachide, présente malgré tout régulièrement sur les tables, semble avoir résisté.

Par contre, les migrants bamiléké ont conservé, eux, une bonne partie de leur identité. Leur façon de préparer est liée à leur manière de manger qui a à voir avec leur corps culturel. Lorsqu'on entre dans la cuisine d'un foyer bamiléké, on observe généralement la présence de beaucoup d'ustensiles artisanaux traditionnels. Ce sont, par exemple, des marmites en bois pour mélanger les ingrédients lors de la préparation de certains mets, des cuillères en bois, des spatules en bois de toutes sortes et, surtout, un coin pour le feu de bois. Car la façon de préparer les mets exige un feu naturel fait à partir de bois. Cela permet de conserver la saveur des mets et offre pour une bonne cuisson.

D’après ce qui précède, on peut déjà avancer, de manière générale, que la modernité combinée à l'urbanisation a entraîné la modification des habitudes alimentaires (confection des mets), notamment la qualité de l'huile rouge consommée, bien que 
cela ne se fasse pas sans résistance. Aussi note-on l'échange et la mobilité de pratiques culinaires. Par exemple, le koki des Bamiléké est préparé au-delà de ses frontières d'origine (l'ouest Cameroun) ; le N'Fian owondo des Béti est aussi préparé par les migrants bamiléké.

Les migrants sont poussés à la diversité culinaire, ce qu'on appelle "l'hétéroculinaire". Cela est révélateur non pas de l'abandon des traditions ou des ethno-codes culinaires, mais montre plutôt le dynamisme, la capacité à s'adapter à des milieux nouveaux. C'est un mouvement qui est accéléré par l'urbanisation, un processus qui implique une mutation importante des dimensions spatiale et temporelle de la cuisine.

Il est à noter que les quantités des différents ingrédients qui entrent dans la composition de ces mets sont déterminées de façon empirique; la ménagère sent d'elle-même comment doser pour obtenir le résultat escompté.

\section{Dimensions spatiale et temporelle}

La cuisine se décline en effet dans le temps. On cuisine plusieurs fois par jour et il faut un moment pour préparer chaque type de plat. Il faut un temps précis pour préparer chaque met. La cuisine change aussi selon les périodes de l'année. Chez les Béti, par exemple, pendant la saison des pluies, les feuilles de manioc reçoivent trop d'eau, alors qu'en saison sèche, c'est le contraire. Il faut donc s'adapter. Lors des nombreuses festivités, la cuisine n'est pas la même non plus.

L'urbanisation introduit, cependant, des éléments nouveaux dans cette dimension culinaire. De plus en plus, on ne prépare plus certains plats à la maison, comme le poisson braisé, la bouillie de maïs ou de farine. Ces types de plats sont proposés par la cuisine de rue. Depuis l'instauration des journées de travail continues, cette cuisine de rue a pris de l'ampleur.

La ville pousse de plus en plus les migrants à la cuisine dite "pratique", c'est-à-dire celle qui est facile à réaliser. Il s'agit du riz sauté, des omelettes, etc. En ville, on mange ce qu'on peut acheter et non ce que sa culture produit, par conséquent, si on ne mange pas ce que sa culture produit, cela veut dire qu'on ne mange plus ce qu'on veut mais ce qu'on peut préparer facilement. La cuisine de rue propose des produits "prêts à manger" adaptés au niveau du goût, du prix et du gain de temps aux exigences de la ville. On voit donc les marchands sillonner les rues et vendre ces nourritures issues de la cuisine ambulante. En résumé, si la cuisine locale des migrants résiste au vent de la modernité, dans le souci de s'adapter aux nouvelles contraintes, aux nouveaux goûts, aux nouvelles saveurs, elle est obligée de changer, d'innover. 


\section{Innovations culinaires : résultat de l'appauvrissement de la cuisine domestique}

L'art culinaire se construit à travers des normes et beaucoup de pratique. Il est lié à un savoir-faire et à une touche personnelle où s'exprime le talent du cuisinier ${ }^{(7)}$. La pratique doit néanmoins respecter un équilibre entre tradition et innovation. Face au développement d'une approche originale du régime ou d'une hygiène alimentaire pour lutter de façon préventive contre le surpoids ou l'obésité, les pratiques alimentaires sont de moins en moins intimement liées à la structure familiale et à l'existence de liens familiaux - bref à la culture. Pour ce qui est de la cuisine, elle reste un marqueur identitaire fondamental. Malheureusement, les migrants se sentent menacés dans leur culture par la cuisine exotique, qui constitue un espace où les normes traditionnelles se relâchent, même si, par la découverte de normes et codes, elle permet de mieux rencontrer l'autre.

Au Cameroun, la modernité combinée à l'urbanisation ne cesse d'entraîner la modification des habitudes alimentaires. Cette modification des habitudes alimentaires est responsable de certaines maladies : anomalies respiratoires, affections de la vésicule, certains cancers..., obésité et maladies cardiovasculaires.

En fait, les migrants sont des êtres paradoxaux; ils sont tiraillés entre le naturel et le culturel, car la cuisine elle-même est à l'articulation du naturel et du culturel. De plus en plus, ils trouvent que la nourriture qui est au coin de la rue peut être culturellement comestible. La cuisine domestique s'en trouve appauvrie. En effet, la cuisine domestique en ville tend à descendre dans la rue pour s'adapter aux contraintes de rapidité et de simplicité propres à la cuisine collective. La cuisine familiale, qui est saine et a du goût, prend, elle, beaucoup de temps. Les mets traditionnels se prêtent mal, en effet, à une préparation rapide et à une consommation sur le pouce. Or la cuisine traditionnelle, qui exige beaucoup d'ingrédients, est aujourd'hui envahie par les bouillons Kub, les arômes et autres exhausteurs de goût industriels. Cette ouverture à ces condiments modernes nous conduit à nous interroger sur l'impact de cette cuisine sur la santé des populations urbaines.

Même si la sauce a été préparée avec tous les ingrédients traditionnels, désormais les migrants y ajoutent quelques Kub, qui sont devenus de véritables symboles. Peut-être ces Kub donnent du goût au plat, mais il est important de chercher à savoir si ce mélange d'ingrédients peut avoir des effets secondaires. En effet, ces arômes qui apportent une saveur standardisée ne sont pas sans danger. Selon les spécialistes, le glutamate de sodium qui entre dans leur composition est toxique à haute dose et, en plus, sa valeur nutritionnelle est faible. Pour nous, il serait abusif 
de dénier à ces arômes toute valeur nutritive. Mais il est tout aussi évident qu'ils sont loin de rivaliser avec les condiments traditionnels qui ont une fonction d'alicament, par exemple le kanwa qui joue un rôle préventif contre les éventuelles attaques des vers intestinaux. L'évolution de la cuisine est une bonne chose, puisqu'elle contribue à diversifier le contenu du plat. Mais il faut savoir gérer le mélange (notamment des huiles) si l'on veut éviter des maladies en relation avec des nourritures issues des cuisines compliquées.

\section{L'huile de palme rouge : un facteur de l'évolution des techniques culinaires}

L'huile rouge est une huile de palme non raffinée, obtenue par pressage des fruits du palmier à huile (Elaeis guineensis). Environ $80 \%$ de sa production est destinée à l'alimentation humaine. Elle est utilisée dans la préparation de plusieurs spécialités comme le koki, le malaxé... et, bien sûr, l'okok. Selon certaines enquêtées, une cuillère à soupe d'huile rouge par jour permet de couvrir les besoins quotidiens d'un enfant. Elle représente le produit végétal le plus riche en béta carotène, précurseur de la vitamine A, micronutriment essentiel. Elle est riche en acides gras, en lipides liposolubles, en vitamines $\mathrm{A}$ et $\mathrm{E}$ et permet de prévenir de nombreuses maladies cardiovasculaires, cancéreuses et visuelles ${ }^{(8)}$. Or, en ville, elle est décolorée, ce qui lui retire son intérêt nutritionnel. Au regard des différentes recettes des Béti et des Bamiléké, il ressort que la nature semble être le facteur primordial et facilitateur de l'appartenance à un corps culturel, qui se meut à la fois dans la diversité et dans la particularité. Les pommes de terre, par exemple, qui reviennent plusieurs fois (sous différentes formes) dans les recettes, justifient leur capacité à de multiples préparations. Au cours des investigations, on a constaté que l'huile rouge fait partie des ingrédients de près de $100 \%$ des mets traditionnels. Plusieurs recettes sont faites avec l'huile rouge.

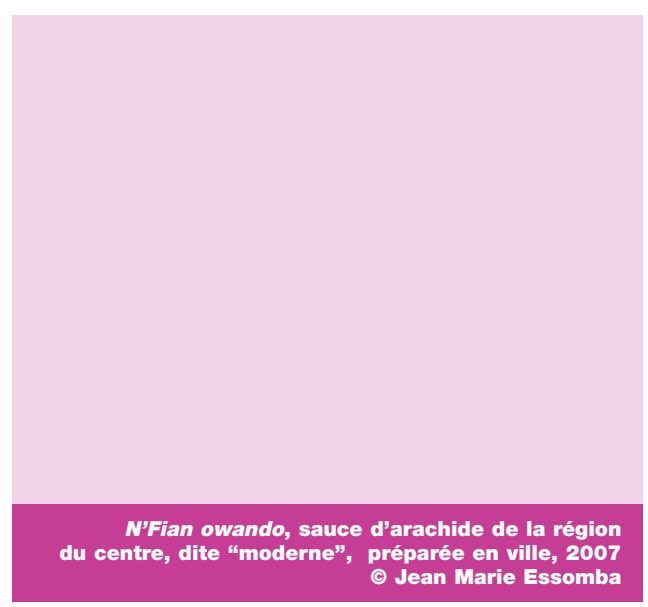


Mais son utilisation pour la confection des mets connaît de plus en plus de modifications, surtout de par son caractère blanchissant. En fait, au village, elle était autrefois utilisée dans son état naturel, c'est-à-dire non chauffée ou légèrement chauffée (juste pour liquéfier davantage). Pour cela, elle conservait toujours sa couleur originelle. Mais le constat est différent aujourd'hui. On se rend compte que l'huile rouge est de plus en plus chauffée par ceux qui vivent en ville pour la cuisson des différents mets, comme les légumes sautés, les pommes pilées ou l'okok, pour ne citer que ceux-là. Or l'huile rouge perd ses qualités lorsqu'elle est surchauffée. Elle contient, par ailleurs, du cholestérol, qui se place dans la liste des premières molécules contribuant au développement de l'obésité. Le développement de cette molécule entraîne la synthèse de certains corps comme les prostaglandines, impliquées dans certains états pathologiques. Cet état de fait est encouragé ou accompagnée par le désordre alimentaire (attachement au mode alimentaire importé) au sein des familles.

Cette cuisson de l'huile rouge se fait sous plusieurs prétextes : par conformisme ou parce qu'on veut cuisiner avec une huile plus légère et/ou avec une huile ayant la couleur de celles présentes dans des bouteilles bien scellées

que, le plus souvent, on n'a pas assez d'agent pour s'acheter. Pour respecter, l'usage de l'huile rouge dans certains mets, des citadins la chauffent juste un peu pour faire cuire les oignons. Dans certaines familles plus ou moins sensibilisées aux conséquences de la surconsommation de lipides, l'huile (l'huile rouge, en particulier) n'est plus abondamment utilisée ; même pour les mets qui en demandent en grande quantité (koki, pilé...) pour leur bonne réalisation.

Par ailleurs, la modification des habitudes alimentaires n'a pas lieu seulement au niveau de la qualité de l'huile rouge consommée. Elle se fait aussi au niveau de la composition - c'est-à-dire des ingrédients et autres - des mets. C'est le cas, par exemple, de sauces qui se font avec de nouveaux ingrédients tels la tomate, les condiments verts, l'oignon, le bouillon Kub. Le sack (couscous maïs) et le kouakekou (macabo rapé) sont de plus en plus présentés dans des emballages en polyéthylène (plastique). Le plastique, de par sa caractéristique de produit non dégradable, 
présente un danger pour la santé humaine car il contribue au développement des cancers. De plus, d'un point de vue gustatif, un couscous emballé dans des feuilles de bananier s'apprécie de manière très différente.

Les migrants ont également intégré de nouveaux aliments dans leur régime alimentaire. Le haricot rouge est accessible sur le marché à tout moment de l'année et son prix est abordable et ne varie pas beaucoup. Le riz a un prix assez bas, permet de nourrir une grande quantité de personnes et, en plus, il se trouve dans tous les points de vente de produits alimentaires. Les pâtes alimentaires sont faciles à cuisiner, accessibles à toutes les bourses et faciles à trouver sur le marché. Les divers poissons de mer se retrouvent également sur le marché et sont à des prix abordables selon la bourse de chaque famille. Ils offrent une alternative à la raréfaction des poissons d'eau douce.

\section{Les modifications des mets traditionnels}

Nkpwem : en ville, il est peu fréquent. On y ajoute du sucre. Les habitudes de la ville ont fait qu'on n'arrive plus à manger des plats complètements fades.

Mbwet : fréquent. La pâte faite de maïs frais écrasé est remplacée par de la farine de maïs sec qui se conserve plus longtemps, le maïs frais étant périodique sur le marché. Le jus de canne à sucre est remplacé par le sucre industriel en morceaux ou en poudre. Il est plus facile de se procurer du maïs sec sur le marché et de le moudre. Par ailleurs, le sucre industriel est facilement accessible, donc le travail est moins pénible.

N'Fian wondo: très fréquent. On ne met plus seulement du poisson séché, mais aussi toutes sortes de viandes. On y a également ajouté d'autres condiments, comme le céle-

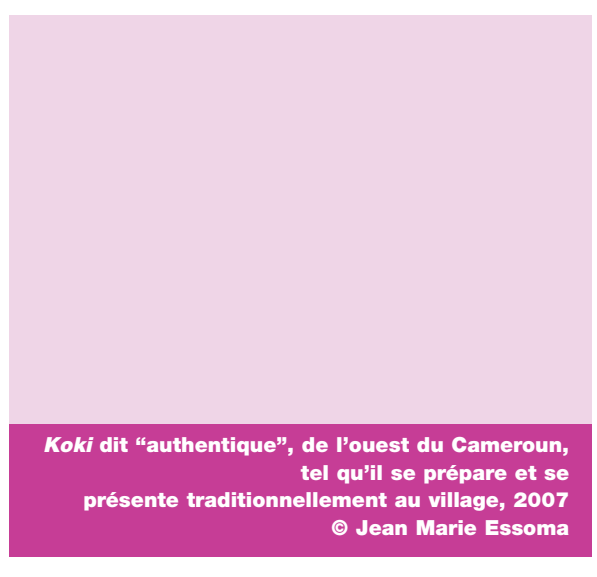
ri, qui peut facilement être trouvé en ville à la place de l'ossim devenu rare ou les concentrés d'aromes appelés "Kub", qui donnent rapidement un goût succulent à la sauce. Avant, on pêchait soi-même son poisson et on le faisait fumer; il avait un goût particulièrement bon. Maintenant, 
le poisson séché en ville n'a plus le même goût. En plus, certains condiments comme l'ossim, qui poussent dans les jardins des cases, deviennent rares parce que leur culture à grande échelle est problématique.

Nnam ngoan : c'est un mets rare, consommé seulement dans de grandes occasions. On y met maintenant toutes sortes de viandes, même des viandes blanches, des cufs durs, des oignons écrasés. On recherche rapidement des goûts de plus en plus exquis. Cependant, ce mets devient rare en ville, parce qu'il nécessite un doigté pour mettre la pâte dans les feuilles de bananier, et les jeunes filles qui n'ont pas eu le temps d'apprendre à le faire ne s'aventurent pas souvent dans sa confection.

Nnam wondo : il est rare, consommé seulement dans de grandes occasions. Les modifications ne sont pas majeures dans sa composition en ville. On ne trouve pas facilement des feuilles de bananier et la technique d'emballage nécessite un certain apprentissage.

Efirgui zoan : peu fréquent. On ajoute du sucre pour camoufler l'amertume des aubergines. Les gens préfèrent maintenant des plats avec des goûts bien plus doux.

Domba : il est rare, consommé seulement en de grandes occasions. On ajoute déjà beaucoup de condiments de la ville comme l'ail, le poireau et même les Kub. La technique d'emballage est délicate et le poisson se fait rare dans les rivières : seul le poisson de mer est aisément accessible en ville. Les condiments, comme le domba traditionnel, sont rares, donc chers sur le marché.

Foass : ce mets, fait à base de larves d'hannetons, est devenu rare, ainsi que l'huile de l'adjap, parce que les jeunes générations n'ont pas pérennisé la technique d'extraction de cette huile.

Eba fon : il est peu fréquent et les modifications ne sont pas majeures dans la technique d'emballage.

Sa fono : il est peu fréquent et les modifications ne sont pas majeures. On note tout de même beaucoup de travail et le temps de cuisson reste toujours long pour la ville. Sanga : il est fréquent. On ajoute du sucre qui sert à donner du goût, car le plat est fade.

Minkôn : il est rare. On ne note pas de modifications majeures. Les chenilles sont rares, l'élaboration du plat est laborieuse et, surtout, les gens trouvent de plus en barbare de manger des chenilles en ville.

Nfian essouk : il est rare en ville. Les modifications ne sont pas majeures. Ce plat nécessite beaucoup de travail et le temps de cuisson est long.

On constate que les mets traditionnels sont très peu préparés par les migrants pour des raisons notamment techniques. Cependant, les migrants ont incorporé dans leurs habitudes alimentaires les mets d'autres tribus et même certains plats d'origine urbaine. 


\section{Conclusion}

Au terme de cette réflexion, on note des mutations culinaires chez les migrants. Cela peut se traduire par ce que Muzele Munzimi ${ }^{(9)}$ a appelé des "homologies structurelles" de la gastronomie des différents corps culturels. En tant que lieu de rencontre des produits d'ici et d'ailleurs (le riz est importé, les poulets congelés), des produits des petites exploitations (la majorité) et des industries (huile de palme rouge), la cuisine est un lieu de rencontre et d'échanges. Les nouveaux aliments issus des échanges culinaires sont une source d'invention, mais peuvent être aussi source d'inquiétudes en terme de risques sanitaires. Dans un contexte de mobilité, la cuisine est en constante mutation. Les migrants s'affirment et affirment leur culture d'appartenance à travers la cuisine et le repas partagé, parce que les plats traditionnels sont perçus comme une "nourriture de l'âme ${ }^{(10) !}$. Parce que la cuisine est considérée comme un lieu de dynamiques et de négociations de rapports ambivalents et contradictoires ${ }^{(11)}$, plus les migrants passent de temps en ville plus ils pratiquent une cuisine qu'ils jugent bonne pour le goût et pour le corps. C'est dire que l'héritage culinaire est soumise à l'épreuve de la diversification culturelle et de l'ouverture à la mobilité. Dès lors, on comprend pourquoi les pratiques culinaires se transmettent de moins en moins chez les migrants, comme l'ont aussi observé Poulain et Corbeau ${ }^{(12)}$ dans le cadre d'études similaires en Europe.

\section{Notes}

1. Annie Hubert et Geneviève Cazes-Valette Hubert, "Introduction", in Faire la cuisine : analyses pluridisciplinaires d'un nouvel espace de modernité, Toulouse, Cahiers de l'OCHA, 2006.

2. François Sigaut, "Le pain, histoire alimentaire, histoire technique", in Alimentation, technique et innovations dans les régions tropicales, sous la direction de José Muchnik, Paris, L'Harmattan, 1999.

3. Système francophone d'information agricole (SYFIA), L'Afrique, côté cuisines. Regards africains sur l'alimentation, Paris, Syros, 1994.

4. E. Messer, "Anthropological Perspective on Diet", Annuel Review of Anthropology, vol. 13, 1984, p. 205-249; I. Garine, The Sociocultural Aspects of Nutrition,.Ecology of Food and Nutrition, vol.1, n², mars 1972, New York, London, Paris, pp.143-164 ; C. Fischler, L'Homnivore. Le goût, la cuisine et le corps. Paris Odile Jacob, 1990.

5. C. Perlès, "Les origines de la cuisine. Lacte alimentaire dans l'histoire de l'homme", in La Nourriture. Pour une alimentation bio culturelle de l'alimentation, Communication, $\mathrm{n}^{\circ} 31,1979$, pp. 4-14.

6. J. Goody, Cuisines, cuisine et classes, Paris, Centre Georges Pompidou, 1984 ; C. Lévi- Strauss, "Le triangle culinaire", L'arc, n²6, 1965, pp. 19- 29.

7. V. Lanouguère-Bruneau, "Des normes à boire et à manger. Production, transformation et consommation des normes alimentaires", in Journal des anthropologues, $\mathrm{n}^{\circ}$ 106-107, 2006.

8. Adelphe L. Pousseu, "Production des semences de palmier à huile (Elaeis guineensis Jacq) sélectionné", rapport de stage DESS UYI, 2007.

9. Muzele Munzimi, Les pratiques de sociabilité en Afrique. Les mutations culinaires chez les Ambuun, Publibook, 2006.

10. Annie Hubert, "Cuisine et politique : le plat national existe-t-il ?", in Révolution dans les cuisines, Revue des sciences sociales, $\mathrm{n}^{\circ} 27,2000$.

11. C. Crenn, "Pratiques alimentaires et sanitaires des Marocains comme lien social dans le vignoble", Actes du colloque de l'AISLF: l'individu et le lien social, (sous la direction de Jean Pierre Corbeau), université de Tours, mars 2005.

12. J.-P. Corbeau et J.-P. Poulain, Penser l'alimentation : entre imaginaire et rationalité, Paris, Privat, 2002. 